\title{
Emerging Role of Notch in Stem Cells and Cancer
}

\author{
Zhiwei Wang, Yiwei Li, Sanjeev Banerjee, and Fazlul H. Sarkar \\ Department of Pathology, Karmanos Cancer Institute, Wayne State University School of Medicine, \\ Detroit, Michigan
}

\begin{abstract}
The Notch signaling pathway is known to be responsible for maintaining a balance between cell proliferation and death and, as such, plays important roles in the formation of many types of human tumors. Recently, Notch signaling pathway has been shown to control stem cell self renewal and multi-potency. As many cancers are thought to be developed from a number of cancer stem-like cells, which are also known to be linked with the acquisition of epithelial-mesenchymal transition (EMT); and thus suggesting an expanding role of Notch signaling in human tumor progression.
\end{abstract}

\section{Notch pathway}

Notch signaling is involved in cell proliferation and apoptosis which affects the development and function of many organs. Notch genes encode proteins which can be activated by interacting with a family of its ligands. Upon activation, Notch is cleaved, releasing intracellular domain of the Notch (ICN) through a cascade of proteolytic cleavages by the metalloprotease tumor necrosis factor- $\alpha$-converting enzyme (TACE) and $\gamma$-secretase. The first cleavage is mediated by TACE, which cleaves the receptor in the extracellular domain. The released extracellular domain is then trans-endocytosed by the ligand-expressing cell. The second cleavage caused by the $\gamma$-secretase activity of a multi-protein complex consisting of presenilin, nicastrin, etc. releasing the intracellular fragment of Notch (ICN) which is then ready to be translocated into the nucleus for transcriptional activation of Notch target genes $(1,2)$. Therefore, inhibiting $\gamma$-secretase activity could prevent the cleavage of the Notch receptor, thus blocking Notch signal transduction.

In the absence of ICN cleavage, transcription of Notch target genes is inhibited by a repressor complex mediated by the CSL (CBF1, Suppressor of Hairlesss or Lag-1). When ICN enters the nucleus, it recruits transcription activators to the CSL complex and converts it from a transcriptional repressor into an activator, which activates the Notch target genes $(1,2)$. To date, four vertebrate Notch genes have been identified: Notch-1-4. In addition, five ligands, such as D11-1, Dll-3, Dll-4, Jagged-1 and Jagged-2, have been found in mammals. A few Notch target genes have also been identified, some of which are dependent on Notch signaling in multiple tissues, while others are tissue specific. Notch target genes include the Hes-1 (Hairy

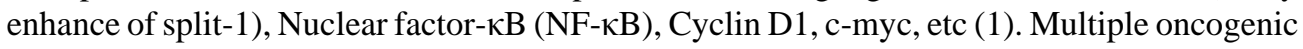
pathways, such as NF-kB, Akt, Sonic hedgehog (Shh), mammalian target of rapamycin

\footnotetext{
*Requests for reprints: Fazlul H. Sarkar, Ph.D. Department of Pathology, Karmanos Cancer Institute, Wayne State University School of Medicine, 9374 Scott Hall, 540 E Canfield, Detroit, MI 48201. Tel: 313-576-8327; Fax: 313-576-8389; E-mail: E-mail: fsarkar@med.wayne.edu.

Conflict of Interest Statement: NO

Publisher's Disclaimer: This is a PDF file of an unedited manuscript that has been accepted for publication. As a service to our customers we are providing this early version of the manuscript. The manuscript will undergo copyediting, typesetting, and review of the resulting proof before it is published in its final citable form. Please note that during the production process errors may be discovered which could affect the content, and all legal disclaimers that apply to the journal pertain.
} 
(mTOR), Ras, Wnt, epidermal growth factor receptor (EGFR) and platelet-derived growth factor (PDGF) signaling have been reported to cross-talk with Notch pathway and, thus it is generally believed that the cross-talk between Notch and other signaling pathways plays important roles in cancer stem cells and tumor aggressiveness (3-9) as discussed below.

\section{Stem Cells and Cancer Stem-like Cells}

Recent results have indicated that Notch signaling pathway contributes to cancer progression by activating transcription factors that promote cell survival, motility, and tumor angiogenesis. Recently, many reports describing molecular connections between Notch regulated transcription factors and pathways known to control stem cell function, further suggesting a new mechanism claiming that Notch may drive tumor growth through the generation or expansion of tumor-initiating cells or cancer stem-like cells (10-15).

Stem cells are characterized by their capacity to self-renewal, and differentiate into the full spectrum of cells forming a particular organism or tissue. Stem cells consists three major types: embryonic, germinal, and somatic (16). The inner cell mass of the blastocyst generates embryonic stem cells. The embryonic stem cells have the capacity to generate any cell types in the mature organism, and also have unlimited capacity to replicate. Germinal stem cells come from the germinal layer of the embryo and they differentiate to generate specific organs. Somatic stem cells have the capacity to self-renew and differentiate into many types of cells that are the characteristics of a specific organ or tissue (16).

Stem cells often stay at locations which are called stem cell niches. Specifically, stem cell niches are defined as particular locations or microenvironments that maintain the combined properties of stem cell self-renewal and multi-potency. There are three kinds of stem cell niches such as simple niches, complex niches, and storage niches (17). Simple niches are defined as a specific location in a tissue where stem cells can reside for an indefinite period of time and produce progeny cells while self-renewing. In general, a stem cell is associated with a permanent partner cell through an adherent junction. The stem cells divide to release another stem cell and a differentiating daughter cell. Complex niches mean that two or more different stem cells are supported by one or more different partner cells. Their activity is coordinately regulated to generate multiple progeny of cells by niche regulatory signals. Storage niches are locations that quiescent stem cells are maintained in a niche until activated by external signals to divide and migrate (17).

A combination of genetic and molecular analyses has identified many factors that support stem cell niche that also control stem cell identity. These factors include components of Notch, Wnt, and Sonic hedgehog (Shh) signaling pathway (18-20). It has been suggested that the capability of a tumor to grow and propagate is due to a small subset of cells within the tumor, termed cancer stem-like cells (CSCs). Although the concept of "cancer stem-like cell" was first proposed more then 150 years ago, it has become more attractive recently due to advances in stem cell biology, leading to the identification of these cells from a wide variety of human cancers (21). CSCs have been identified and isolated from tumors of the hematopoietic system, breast, lung, prostate, colon, brain, head and neck, and pancreas (22). CSCs are able to selfrenew, differentiate, and regenerate to a phenotypic cells of the original tumor when implanted into the severe combined immunodeficient mouse (22). The concept of CSCs have generated considerable attention in recent years, which is likely to provide clear understanding of tumor biology, and for designing novel therapy targeted toward these cells for the complete eradication of tumor growth. 


\section{Notch pathway in stem cells}

It has been reported that altered Notch signaling affects the function of a variety of mammalian stem cells such as hematopoietic, intestinal, and skin stem cells, and intestinal stem cells in Drosophila and germ stem cells in C. elegans $(17,19,23)$. Recently, ohlstein et al. reported that intestinal stem cells (ISCs) in adult Drosophila midgut containing high levels of cytoplasmic Delta-rich vesicles activate the canonical Notch pathway and down-regulate Delta within their daughters, a process that programs these daughters to become enterocytes. However, ISCs expressing little vesiculate Delta, or being impaired in Notch signaling, specify their daughters to become enteroendocrine cells. Thus, ISCs control daughter cell fate by modulating Notch signaling over time, suggesting that ISCs actively coordinate cell production with local tissue requirements regulated by Notch signaling pathway (24).

The Drosophila germline stem cells (GSCs) reside in a somatic cell niche. Ward et al. showed that Notch activation can induce the expression of niche-cell markers even in an adult fly. Overexpression of Delta in the germline, or activated Notch in the somatic cells, results in extra niche cells, up to 10-fold over the normal number. In turn, these ectopic niche cells induce ectopic GSCs (25). In addition, Notch signaling is required for hypoxia promoting the undifferentiated cell state in various stem and precursor cell populations. Hypoxia blocks neuronal and myogenic differentiation in a Notch-dependent manner. Hypoxia activates Notchresponsive promoters and increases expression of Notch directed downstream genes such as Hes-1 and Hey-2. The ICN interacts with HIF- $1 \alpha$, and HIF- $1 \alpha$ is recruited to Notch-responsive promoters upon Notch activation under hypoxic conditions. These data provide molecular insights into how Notch controls the cellular differentiation status (26) and the maintenance of stem cells.

Notch signaling is also critical for the maintenance of undifferentiated stem and progenitor cell populations in the mammalian intestinal crypt and also influences differentiation of mature enterocytes (10). Wilson et al. suggested that many of the general Notch functions such as stem cell gate keeper, influencing binary cell fate decisions or induction of terminal differentiation processes exists in invertebrates and self-renewing organ systems of mammals (10). In the intestine, Notch plays a gate-keeper function for crypt progenitor cells. Notch also seems to control binary cell fate decisions of cells that have to choose between the secretory and adsorptive lineages, most likely by Notch induced expression of Hes-1 $(10,27)$.

\section{Notch pathway in cancer stem cells}

Cancer stem cells constitute a small subset of cancer cells being a reservoir of self-sustaining cells with the exclusive ability to self-renew and maintain the tumor. These cells are characterized by specific stem cell markers: antigens, molecules and signaling pathways. The pathways that regulate self-renewal and cell fate in these systems are beginning to be elucidated. Transcription factors and molecules associated with oncogenesis, such as Notch, NF- $\kappa B$, B lymphoma Mo-MLV insertion region (Bmi-1), Wnt, Sonic hedgehog pathways, are active only in a small minority of cancer cells although they may play key roles in determining the biological behavior of a tumor (28). Katoh reported that the balance between Wnt-FGFNotch and BMP-Hedgehog signaling networks is important for the maintenance of homeostasis among stem and progenitor cells. Disruption of the stem cell signaling network results in congenital diseases and cancer (29). In addition to pathways such as Wnt, Notch and Hedgehog are known to regulate self-renewal of normal stem cells, while tumor suppressor genes such as PTEN (phosphatase and tensin homolog on chromosome 10) and P53 (tumor protein p53) are also reported to have implications in the regulation of cancer stem cell self-renewal (21).

It has been reported that Notch signaling plays a critical role in normal human mammary development by acting on both stem cells and progenitor cells, suggesting that abnormal Notch 
signaling may contribute to mammary carcinogenesis by deregulating the self-renewal of normal mammary stem cells (30). Dontu et al. observed a 10-fold increase in secondary mammosphere formation upon addition of a Notch-activating DSL peptide, suggesting that Notch signaling can act on mammary stem cells to promote self-renewal. Notch signaling was also found to act on multi-potent progenitor cells, facilitating myoepithelial lineage-specific commitment and proliferation. Phillips et al. reported that cancer stem cells can be identified by phenotypic markers and their fate is controlled by the Notch pathway in breast cancer. Recombinant human erythropoietin receptor increased the numbers of stem cells and selfrenewing capacity in a Notch-dependent fashion by induction of Jagged-1. Inhibitors of the Notch pathway blocked this effect, suggesting the mechanistic role of Notch signaling in the maintenance of cancer stem-like cell phenotype (31). Farnie et al. also provided evidence for breast cancer stem cells, and their studies have consistently shown that stem-like cells and breast cancer initiating populations can be enriched using cell surface makers CD44+/CD24and, as such, these cells showed up-regulated genes including Notch that are known to contribute to cancer stem-like cells characteristics (32).

A small population of cancer stem cells obtained from brain tumors could form neurospheres, which have the capacity for self-renewal, and are able to differentiate into diverse populations including neuronal, astrocytic and oligodendroglial cells when dissociated in single cell suspension $(33,34)$. The small population of stem cells, also termed "side population" (SP), has been found in long-term culture of glioma cell lines such as human U87-MG and U373MG (35). SP stem cells have elevated chemo-resistance because of the high expression levels of drug-transporter proteins such as ABCG2, an ATP-binding cassette half-transporter associated with multi-drug resistance. Furthermore, ABCG2 expression is also associated with proliferation, and the ABCG2 positive cells preferentially expresses several "stemness" genes such as Notch-1 (35).

Since gliomas are the most common tumors of the central nervous system and an important cause of mental impairment and death, emerging research has been intensified for this disease. Increasing body of evidence suggests that gliomas may rise from cancer stem cells sharing with neural stem cells the capacity of self-renewal and multi-potency. Interestingly, Notch signaling has been shown to be involved in promoting the formation of cancer stem cell-like cells in human glioma. Notch signaling is appears to be essential for the maintenance of neural stem cells (NSC), by enhancing the NSC self-renewal and by inhibiting its differentiation into neuronal and glial progenitor (36-38). In addition, Notch signaling prevents nestin degradation during neural stem cell differentiation, by a mechanism that involves ubiquitin-proteosome pathways (39). Recent data also suggest that Notch signaling can directly up-regulate nestin expression in gliomas, and cooperate with K-ras to lead to their expansion within the subventricular zone and retention of proliferation and nestin expression (40). Recently, Zhang et al reported that over-expression of Notch-1 in SHG-44 glioma cells promoted the growth and the colony-forming activity of these cells. Moreover, over-expression of ICN increased the formation neurosphere-like colonies in the presence of growth factors. These colonies expressed nestin, and these cells could be induced to cell types that expresses specific markers such as neuron, astrocyte, or oligodendrocyte, which is consistent with phenotypes of neural stem cells, suggesting that potential functions of the Notch pathway in the formation of cancer stem cells in human glioma (41). Fan et al. also found that Notch blockade reduced the CD133positive cell fraction almost 5 -fold and totally abolished the side population. These data suggested that the loss of tumor-forming capacity could be due to the depletion of stem-like cells. Notch signaling levels were higher in the stem-like cell fraction, providing a potential mechanism for their increased sensitivity to the inhibition of this pathway. They also observed that apoptotic rates following Notch blockade were almost 10-fold higher in primitive nestinpositive cells as compared with nestin-negative cells. Stem-like cells in brain tumors thus seems to be selectively vulnerable to agents that will inhibit the Notch pathway (42). 
Moreover, Jagged-2, a Notch ligand, was found to be over-expressed in the leukemic stem cells (LSC) samples. DAPT, an inhibitor of gamma-secretase, a protease that is involved in Jagged and Notch signaling, inhibits LSC growth as documented by colony formation assays (43). Taken together, these results suggested that Notch pathway plays an important role in cancer stem-like cells and thus Notch signaling pathways appears to be a legitimate target for cancer therapy.

\section{Notch pathway in multiple cancer and cancer stem cells}

Emerging evidence clearly suggest that cancers can grow from cancer stem cells. In cancer stem cells, Notch pathway is believed to be deregulated, leading to uncontrolled self-renewal of cancer stem cells which generate tumor mass. Indeed, Notch gene is abnormally activated in many human malignancies. It has been reported that the Notch signaling network is frequently deregulated in human malignancies with up-regulated expression of Notch receptors and their ligands in cervical, lung, colon, head and neck, renal carcinoma, acute myeloid, Hodgkin and Large-cell lymphomas and pancreatic cancer $(1,2,9,44)$. However, in a limited number of tumor types, including human hepatocellular carcinoma, skin and small lung cancer, Notch signaling is anti-proliferative rather than oncogenic $(1,2)$, suggesting that further molecular and mechanistic studies are warranted to ascertain the specific role of Notch family of proteins in cancer stem-like cells. High-level expression of Notch-1 and Jagged-1 was found to be associated with poor prognosis in breast cancer and prostate cancer. The tumor expressing high levels of Jagged-1 protein in patients had a worse outcome than those with tumors expressing low levels $(45,46)$. Recent reports have shown that Notch-1 expression regulates cell death through both apoptosis and cell cycle pathways in erythroleukemia cells with regulation of c-Jun N-terminal kinase (JNK), Bcl-xL, p2 $1^{\text {cip } 1, ~ p 27 k i p 1, ~ N F-\kappa B ~ a n d ~ t h e ~}$ retinoblastoma protein $\mathrm{Rb}(2,47)$. The growing body of literature strongly suggests the biological relevance of Notch signaling in cancer cell growth, invasion and metastasis, which further suggest that the inactivation of Notch signaling by novel approach could be useful for cancer therapy. Interestingly, we found that Notch is much more activated in gemcitabine resistant L3.6pl pancreatic cancer cells which showed typical characteristics of EMT phenotype as reported previously (48). Moreover, we have previously found that a natural product, curcumin is a potent agent in the down-regulation of Notch signaling in pancreatic cancer (49); however the mechanistic role and the consequence of the down-regulation of Notch signaling in EMT-type cells remains to be elucidated.

Current cancer therapeutic strategies based on tumor regression may target and kill differentiated tumor cells, which constitute the bulk of the tumor, while sparing the rare cancer stem cell population. The cancer stem cell model suggests that the design of new cancer therapeutics may require the targeting and elimination of cancer stem cells. Therefore eradicating cancer stem cells is an important goal in curing cancer, and thus the Notch pathway is considered an attractive target for treatment of cancer because Notch-targeting will not only kill differentiated cancer cells but could also kill cancer stem cells. Moreover reducing Notch activity in cancer stem cells could promote their differentiation, leading to reduce their ability to repopulate the cells in forming tumor mass. These hypotheses must be molecularly and mechanistically tested in multiple tumor system in order to fully appreciate the role of Notch signaling in cancer stem cells. Since Notch signaling is activated via the activity of $\gamma$-secretase; therefore inhibitors of $\gamma$-secretase viewed as novel target for therapy and, as such, some inhibitors are being tested in Phase I clinical trials, which underscores the importance of Notch signaling for cancer therapy. These results clearly suggest that inactivation of Notch signaling by novel approaches is likely to have a significant impact in cancer therapy. However, it is imperative to design new strategies based upon molecular understanding of the Notch and other signaling pathways that controls the biology of self-renewal and survival capacity of cancer stem-like cells that are reminiscent of EMT phenotype. 


\section{Acknowledgments}

We apologize to those colleagues whose work could not be cited here due to space limitations. The authors' work cited in this review was funded by grants from the National Cancer Institute, NIH (5R01CA101870) to F.H.S. and Department of Defense Postdoctoral Training Award W81XWH-08-1-0196 (Zhiwei Wang) and also partly supported by a subcontract award (F.H.S.) from the University of Texas MD Anderson Cancer Center through a SPORE grant (1P20-CA010193-01) on pancreatic cancer awarded to James Abbruzzese.

\section{References}

1. Miele L. Notch signaling. Clin Cancer Res 2006;12:1074-9. [PubMed: 16489059]

2. Miele L, Miao H, Nickoloff BJ. Notch signaling as a novel cancer therapeutic target. Curr Cancer Drug Targets 2006;6:313-23. [PubMed: 16848722]

3. Mungamuri SK, Yang X, Thor AD, Somasundaram K. Survival signaling by Notch1: mammalian target of rapamycin (mTOR)-dependent inhibition of p53. Cancer Res 2006;66:4715-24. [PubMed: 16651424]

4. Nair P, Somasundaram K, Krishna S. Activated Notch1 inhibits p53-induced apoptosis and sustains transformation by human papillomavirus type 16 E6 and E7 oncogenes through a PI3K-PKB/Aktdependent pathway. J Virol 2003;77:7106-12. [PubMed: 12768030]

5. Nakamura T, Tsuchiya K, Watanabe M. Crosstalk between Wnt and Notch signaling in intestinal epithelial cell fate decision. J Gastroenterol 2007;42:705-10. [PubMed: 17876539]

6. Nickoloff BJ, Qin JZ, Chaturvedi V, Denning MF, Bonish B, Miele L. Jagged-1 mediated activation of notch signaling induces complete maturation of human keratinocytes through NF-kappaB and PPARgamma. Cell Death Differ 2002;9:842-55. [PubMed: 12107827]

7. Osipo C, Golde TE, Osborne BA, Miele LA. Off the beaten pathway: the complex cross talk between Notch and NF-kappaB. Lab Invest 2008;88:11-7. [PubMed: 18059366]

8. Rangarajan A, Syal R, Selvarajah S, Chakrabarti O, Sarin A, Krishna S. Activated Notch1 signaling cooperates with papillomavirus oncogenes in transformation and generates resistance to apoptosis on matrix withdrawal through PKB/Akt. Virology 2001;286:23-30. [PubMed: 11448155]

9. Wang Z, Banerjee S, Li Y, Rahman KM, Zhang Y, Sarkar FH. Down-regulation of Notch-1 inhibits invasion by inactivation of nuclear factor- $\{$ kappa $\}$ B, vascular endothelial growth factor, and matrix metalloproteinase-9 in pancreatic cancer cells. Cancer Res 2006;66:2778-84. [PubMed: 16510599]

10. Wilson A, Radtke F. Multiple functions of Notch signaling in self-renewing organs and cancer. FEBS Lett 2006;580:2860-8. [PubMed: 16574107]

11. Charafe-Jauffret E, Monville F, Ginestier C, Dontu G, Birnbaum D, Wicha MS. Cancer stem cells in breast: current opinion and future challenges. Pathobiology 2008;75:75-84. [PubMed: 18544962]

12. Peacock CD, Watkins DN. Cancer stem cells and the ontogeny of lung cancer. J Clin Oncol 2008;26:2883-9. [PubMed: 18539968]

13. Fan X, Eberhart CG. Medulloblastoma stem cells. J Clin Oncol 2008;26:2821-7. [PubMed: 18539960]

14. Kakarala M, Wicha MS. Implications of the cancer stem-cell hypothesis for breast cancer prevention and therapy. J Clin Oncol 2008;26:2813-20. [PubMed: 18539959]

15. Scoville DH, Sato T, He XC, Li L. Current view: intestinal stem cells and signaling. Gastroenterology 2008;134:849-64. [PubMed: 18325394]

16. Kakarala M, Wicha MS. Cancer stem cells: implications for cancer treatment and prevention. Cancer J 2007;13:271-5. [PubMed: 17921723]

17. Ohlstein B, Kai T, Decotto E, Spradling A. The stem cell niche: theme and variations. Curr Opin Cell Biol 2004;16:693-9. [PubMed: 15530783]

18. Bray SJ. Notch signalling: a simple pathway becomes complex. Nat Rev Mol Cell Biol 2006;7:67889. [PubMed: 16921404]

19. Joseph NM, Morrison SJ. Toward an understanding of the physiological function of Mammalian stem cells. Dev Cell 2005;9:173-83. [PubMed: 16054025]

20. Keith B, Simon MC. Hypoxia-inducible factors, stem cells, and cancer. Cell 2007;129:465-72. [PubMed: 17482542] 
21. Korkaya H, Wicha MS. Selective targeting of cancer stem cells: a new concept in cancer therapeutics. BioDrugs 2007;21:299-310. [PubMed: 17896836]

22. Tang C, Ang BT, Pervaiz S. Cancer stem cell: target for anti-cancer therapy. FASEB J 2007;21:377785. [PubMed: 17625071]

23. Ohlstein B, Spradling A. The adult Drosophila posterior midgut is maintained by pluripotent stem cells. Nature 2006;439:470-4. [PubMed: 16340960]

24. Ohlstein B, Spradling A. Multipotent Drosophila intestinal stem cells specify daughter cell fates by differential notch signaling. Science 2007;315:988-92. [PubMed: 17303754]

25. Ward EJ, Shcherbata HR, Reynolds SH, Fischer KA, Hatfield SD, Ruohola-Baker H. Stem cells signal to the niche through the Notch pathway in the Drosophila ovary. Curr Biol 2006;16:2352-8. [PubMed: 17070683]

26. Gustafsson MV, Zheng X, Pereira T, et al. Hypoxia requires notch signaling to maintain the undifferentiated cell state. Dev Cell 2005;9:617-28. [PubMed: 16256737]

27. Fre S, Huyghe M, Mourikis P, Robine S, Louvard D, rtavanis-Tsakonas S. Notch signals control the fate of immature progenitor cells in the intestine. Nature 2005;435:964-8. [PubMed: 15959516]

28. Styczynski J, Drewa T. Leukemic stem cells: from metabolic pathways and signaling to a new concept of drug resistance targeting. Acta Biochim Pol 2007;54:717-26. [PubMed: 18080019]

29. Katoh M. Networking of WNT, FGF, Notch, BMP, and Hedgehog signaling pathways during carcinogenesis. Stem Cell Rev 2007;3:30-8. [PubMed: 17873379]

30. Dontu G, Jackson KW, McNicholas E, Kawamura MJ, Abdallah WM, Wicha MS. Role of Notch signaling in cell-fate determination of human mammary stem/progenitor cells. Breast Cancer Res 2004;6:R605-R615. [PubMed: 15535842]

31. Phillips TM, Kim K, Vlashi E, McBride WH, Pajonk F. Effects of recombinant erythropoietin on breast cancer-initiating cells. Neoplasia 2007;9:1122-9. [PubMed: 18084619]

32. Farnie G, Clarke RB. Mammary stem cells and breast cancer--role of Notch signalling. Stem Cell Rev 2007;3:169-75. [PubMed: 17873349]

33. Singh SK, Clarke ID, Terasaki M, et al. Identification of a cancer stem cell in human brain tumors. Cancer Res 2003;63:5821-8. [PubMed: 14522905]

34. Dell'albani P. Stem Cell Markers in Gliomas. Neurochem Res. 2008

35. Patrawala L, Calhoun T, Schneider-Broussard R, Zhou J, Claypool K, Tang DG. Side population is enriched in tumorigenic, stem-like cancer cells, whereas ABCG2+ and A. Cancer Res 2005;65:620719. [PubMed: 16024622]

36. Nakamura Y, Sakakibara S, Miyata T, et al. The bHLH gene hes1 as a repressor of the neuronal commitment of CNS stem cells. J Neurosci 2000;20:283-93. [PubMed: 10627606]

37. Hitoshi S, Seaberg RM, Koscik C, et al. Primitive neural stem cells from the mammalian epiblast differentiate to definitive neural stem cells under the control of Notch signaling. Genes Dev 2004;18:1806-11. [PubMed: 15289455]

38. Hitoshi S, Alexson T, Tropepe V, et al. Notch pathway molecules are essential for the maintenance, but not the generation, of mammalian neural stem cells. Genes Dev 2002;16:846-58. [PubMed: 11937492]

39. Mellodew K, Suhr R, Uwanogho DA, et al. Nestin expression is lost in a neural stem cell line through a mechanism involving the proteasome and Notch signalling. Brain Res Dev Brain Res 2004;151:1323.

40. Shih AH, Holland EC. Notch signaling enhances nestin expression in gliomas. Neoplasia 2006;8:1072-82. [PubMed: 17217625]

41. Zhang XP, Zheng G, Zou L, et al. Notch activation promotes cell proliferation and the formation of neural stem cell-like colonies in human glioma cells. Mol Cell Biochem 2008;307:101-8. [PubMed: 17849174]

42. Fan X, Matsui W, Khaki L, et al. Notch pathway inhibition depletes stem-like cells and blocks engraftment in embryonal brain tumors. Cancer Res 2006;66:7445-52. [PubMed: 16885340]

43. Gal H, Amariglio N, Trakhtenbrot L, et al. Gene expression profiles of AML derived stem cells; similarity to hematopoietic stem cells. Leukemia 2006;20:2147-54. [PubMed: 17039238] 
44. Wang Z, Zhang Y, Li Y, Banerjee S, Liao J, Sarkar FH. Down-regulation of Notch-1 contributes to cell growth inhibition and apoptosis in pancreatic cancer cells. Mol Cancer Ther 2006;5:483-93. [PubMed: 16546962]

45. Dickson BC, Mulligan AM, Zhang H, et al. High-level JAG1 mRNA and protein predict poor outcome in breast cancer. Mod Pathol 2007;20:685-93. [PubMed: 17507991]

46. Santagata S, Demichelis F, Riva A, et al. JAGGED1 expression is associated with prostate cancer metastasis and recurrence. Cancer Res 2004;64:6854-7. [PubMed: 15466172]

47. Jang MS, Miao H, Carlesso N, et al. Notch-1 regulates cell death independently of differentiation in murine erythroleukemia cells through multiple apoptosis and cell cycle pathways. J Cell Physiol 2004;199:418-33. [PubMed: 15095289]

48. Shah AN, Summy JM, Zhang J, Park SI, Parikh NU, Gallick GE. Development and characterization of gemcitabine-resistant pancreatic tumor cells. Ann Surg Oncol 2007;14:3629-37. [PubMed: 17909916]

49. Wang Z, Zhang Y, Banerjee S, Li Y, Sarkar FH. Notch-1 down-regulation by curcumin is associated with the inhibition of cell growth and the induction of apoptosis in pancreatic cancer cells. Cancer 2006;106:2503-13. [PubMed: 16628653] 